\title{
On Adelic Zeta Functions of Prehomogeneous Vector Spaces with a Finitely Many Adelic Open Orbits
}

\author{
Tatsuo Kimura and Takeyoshi Kogiso
}

\section{Introduction}

The two adelic zeta functions $Z_{a}(\omega, \Phi)$ and $Z_{m}(\omega, \Phi)$ for a prehomogeneous vector space (abbrev. P.V.) $(G, \rho, V)$ have no relation in general. For an irreducible case, Professor J. Igusa showed that $Z_{a}=\tau Z_{m}$ with some constant $\tau$ when \# $\left(G_{A} \backslash Y_{A}\right)<\infty$ under the condition (HW) where $Y$ is the open $G$-orbit in $V$ (see Igusa [4]).

In this paper, we shall show that the condition (HW) is not necessary. Moreover, we shall show that the theorem of the same type holds even for simple P.V's and 2-simple P.V.'s of type I. It is known that when $Z_{a}=\tau Z_{m}$ holds, we can generalize Iwasawa-Tate Theory for such P.V.'s and we can have many informations (see T. Kimura [11]).

\section{Contents}

$\S 1$. Basic definitions

$\S 2$. Some sufficient conditions for $Z_{a}=\tau Z_{m}$

§3. Simple P.V.'s with \# $\left(G_{A} \backslash Y_{A}\right)<+\infty$

§4. 2-Simple P.V.'s of Type I with \# $\left(G_{A} \backslash Y_{A}\right)<+\infty$

\section{$\S 1 . \quad$ Basic definitions}

Let $G$ be a connected reductive linear algebraic group and $\rho: G \longrightarrow G L(V)$ a rational representation of $G$ with the open dense $G$-orbit $Y$. In this case, we call a triplet $(G, \rho, V)$ a prehomogeneous vector space (abbrev. P.V.). The complement $S$ of $Y$ is a Zariskiclosed set which is called the singular set of $(G, \rho, V)$. We assume that the isotropy subgroup $H$ of $\rho(G)$ at a point in $Y$ is connected and semisimple. The irreducible components $S_{i}$ of codimension one

Received January 20, 1991. 
are the zeros of some irreducible polynomials $f_{i}(x)(i=1,2, \ldots, r)$. Then $f_{1}(x), f_{2}(x), \ldots, f_{r}(x)$ are algebraically independent relative invariants, i.e., $f_{i}(\rho(g) x)=\chi_{i}(g) f_{i}(x)$ for $g \in G, x \in V$ with some rational characters $\chi_{i}$ of $G$. Moreover any relative invariant rational function $f(x)$ is of the form $f(x)=c \cdot f_{1}(x)^{m_{1}} f_{2}(x)^{m_{2}} \cdots f_{r}(x)^{m_{r}}$ with $\left(m_{1}, m_{2}, \ldots, m_{r}\right) \in \mathbf{Z}^{r}$ and some constant $c$ (see p.60 in (M. Sato and T. Kimura [5])).

Let $k$ be an algebraic number field. We assume that $(G, \rho, V)$ is defined over $k$ and all coefficients of $f_{i}(x)$ are in $k$. We denote by $G_{A}, V_{A}$, etc. the adelization of $G, V$, etc. with respect to $k$. Let $\Omega\left(k_{A}^{\times} / k^{\times}\right)$be the space of quasicharacters of the idele class group $k_{A}^{\times} / k^{\times}$and $\mathcal{S}\left(V_{A}\right)$ the Schwartz-Bruhat space on $V_{A}$.

For $\omega=\left(\omega_{1}, \ldots, \omega_{r}\right) \in \Omega\left(k_{A}^{\times} / k^{\times}\right)^{r}$, we write $\omega(\chi(g))=\omega_{1}\left(\chi_{1}(g)\right)$ $\cdots \omega_{r}\left(\chi_{r}(g)\right)$ and $\omega(f(x))=\omega_{1}\left(f_{1}(x)\right) \cdots \omega_{r}\left(f_{r}(x)\right)\left(g \in G_{A}, x \in Y_{A}=\right.$ $\left.(V-S)_{A}\right)$ for simplicity. Now we define the two adelic zeta-functions $Z_{a}(\omega, \Phi)$ and $Z_{m}(\omega, \Phi)$ of $(G, \rho, V)$.

$$
\begin{aligned}
& Z_{a}(\omega, \Phi)=\int_{G_{A} / G_{k}} \omega(\chi(g)) \sum_{\xi \in Y_{k}} \Phi(\rho(g) \cdot \xi) d_{G_{A}}(g) \\
& Z_{m}(\omega, \Phi)=\int_{Y_{A}} \omega(f(x)) \Phi(x) d_{Y_{A}}(x)
\end{aligned}
$$

$\left(\Phi \in \mathcal{S}\left(V_{A}\right)\right)$

Here $d_{G_{A}}$ is a Haar measure on $G_{A}$ and $d_{Y_{A}}$ is a $G_{A}$-invariant measure on $Y_{A}$ (see the beginning of $\S 2$ ). We take the same convergence factor for $d_{G_{A}}$ and $d_{Y_{A}}$. The role of $Z_{a}(\omega, \Phi)$ is a functional equation based on the adelic Poisson summation formula while $Z_{m}(\omega, \Phi)$ has an Euler product $Z_{m}(\omega, \Phi)=\Pi_{v \in \Sigma} Z_{v}\left(\omega_{v}, \Phi_{v}\right)$ when $\Phi=\otimes_{v \in \Sigma} \Phi_{v}$ where $\Sigma$ denotes the set of places of $k$.

For the absolute convergence of $Z_{m}(\omega, \Phi)$, see p.90 in (T. Ono [13] and F. Sato [9]).

\section{$\S 2$. Some sufficient conditions for $Z_{a}=\tau Z_{m}$}

For simplicity, we assume that $G \subset G L(V)$ and $(G, V)$ is defined over an algebraic number field $k$. Take a $k$-rational generic point $\eta \in$ $Y_{k}=(V-S)_{k}$ and we denote by $H$ the isotropy subgroup of $G$ at $\eta$. Since we assume that $H$ is semisimple, we have $\operatorname{vol}\left(H_{A} / H_{k}\right)<+\infty$ (see A. Borel and Harish-Chandra [12]), and there exists a $G_{A^{-}}$-invariant measure $d_{Y_{A}}$ on $Y_{A}$. Since $H$ is connected, $G_{A} \cdot \eta$ is open in $Y_{A}$. We normalize measures $d_{G_{A}}, d_{H_{A}}$ and $d_{Y_{A}}$ on $G_{A}, H_{A}$ and $Y_{A}$ by 


$$
\begin{array}{r}
\int_{G_{A}} \phi(g) d_{G_{A}}(g)=\int_{G_{A} \cdot \eta} d_{Y_{A}}(x)\left(\int_{H_{A}} \phi(g h) d_{H_{A}}(h)\right) \\
\left(\text { with } x=g H_{A}\right)
\end{array}
$$

for any $\phi \in L^{1}\left(G_{A}\right)$.

Proposition 1-1. We have

$$
\begin{aligned}
& \int_{G_{A} / G_{k}} \omega(\chi(g)) \sum_{\xi \in G_{k} \cdot \eta} \Phi(g \xi) d_{G_{A}}(g) \\
= & \tau \int_{G_{A} \cdot \eta} \omega(f(x)) \Phi(x) d_{Y_{A}}(x)
\end{aligned}
$$

for $\Phi \in \mathcal{S}\left(V_{A}\right)$ where $\tau=\int_{H_{A} / H_{k}} d_{H_{A}}\left(=\operatorname{vol}\left(H_{A} / H_{k}\right)<+\infty\right)$.

Proof. First we observe that $\omega(\chi(\gamma))=1$ for $\gamma \in G_{k}$ and $\omega(f(\eta))=$ 1, i.e., $\omega(\chi(g \gamma))=\omega(f(g \eta))$.

Since $G$ is reductive,the Haar measure $d_{G_{A}}$ is right-invariant, i.e., $d_{G_{A}}(g \gamma)=d_{G_{A}}(g)$.

Now

$$
\begin{aligned}
\text { L.H.S. } & =\int_{G_{A} / G_{k}} \omega(\chi(g)) \sum_{\gamma \in G_{k} / H_{k}} \Phi(g \gamma \eta) d_{G_{A}}(g) \\
& =\int_{G_{A} / H_{k}} \omega(\chi(g)) \Phi(g \eta) d_{G_{A}}(g) \\
& =\int_{G_{A} \cdot \eta} d_{Y_{A}}(x)\left(\int_{H_{A} / H_{k}} \omega(f(g h \cdot \eta)) \Phi(g h \cdot \eta) d_{H_{A}}(h)\right) \\
& =\tau \int_{G_{A} \cdot \eta} \omega(f(x)) \Phi(x) d_{Y_{A}}(x)
\end{aligned}
$$

where $\tau=\int_{H_{A} / H_{k}} d_{H_{A}}$ is a finite number by assumption.

Q.E.D.

Now the following proposition is obvious.

Proposition 1-2. Assume that $Y_{k}=G_{k} \cdot \eta$ and $Y_{A}=G_{A} \cdot \eta$. Then we have $Z_{a}=\tau Z_{m}$.

Proposition 1-3. Let $(G, V)$ and $\left(G^{\prime}, V\right)$ be P.V.'s satisfying $G \subset$ $G^{\prime} \subset G L(V)$ and $Y^{\prime}=Y$. If $Y_{k}=G_{k} \cdot \eta$ and $Y_{A}=G_{A} \cdot \eta$, then we have $Z_{a}^{\prime}=\tau^{\prime} Z_{m}^{\prime}=\frac{\tau^{\prime}}{\tau} Z_{a}$ and $Z_{m}^{\prime}=Z_{m}$. 
Proof. Since $Y^{\prime}=Y$, we have $Z_{m}^{\prime}=Z_{m}$. Since $G \subset G^{\prime}$, we have $Y_{k}=G_{k}^{\prime} \cdot \eta$ and $Y_{A}=G_{A}^{\prime} \cdot \eta$, hence $Z_{a}^{\prime}=\tau^{\prime} Z_{m}$ by Proposition 1-2. Since $Z_{a}=\tau Z_{m}$, we have $Z_{a}^{\prime}=\frac{\tau^{\prime}}{\tau} Z_{a}$.

Q.E.D.

Proposition 1-4. For $\left(G L_{d}, M_{d}\right)$ with a $k$-form $\left(G L_{d}(k), M_{d}(k)\right)$, we have $Y_{k}=G_{k} \cdot I_{d}$ and $Y_{A}=G_{A} \cdot I_{d}$ (hence we have $Z_{a}=\tau Z_{m}$ ).

Proof. Since $Y_{k}=G L_{d}(k)=G_{k}=G_{k} \cdot I_{d}$, and $Y_{A}=\left(G L_{d}\right)_{A}=$ $G_{A}=G_{A} \cdot I_{d}$, we have our assertion by Proposition 1-2. $\quad$ Q.E.D.

Proposition 1-5. Let $G_{o}$ be a connected $k$-split algebraic subgroup of $S L_{d}$ acting on $M_{d}$ as $\rho\left(g_{o}, g_{1}\right) \cdot x=g_{o} x^{t} g_{1}\left(g_{o} \in G_{o}, g_{1} \in G L_{d}, x \in\right.$ $\left.M_{d}\right)$. Then for a P.V. $\left(G_{o} \times G L_{d}, \rho, M_{d}\right)$ with the $k$-form

$$
\left(\left(G_{o}\right)_{k} \times G L_{d}(k), \rho, M_{d}(k)\right),
$$

we have $Z_{a}=\tau Z_{m}$.

Proof. It is clear by Proposition 1-3 and Proposition 1-4.

Q.E.D.

Theorem 1-6 (Igusa [4] with the above Proposition 1-5). Let $(G, \rho, V)$ be an irreducible regular $P . V$. defined over $k$ such that $Y_{A}$ decomposes into a finitely many $G_{A}$-orbits. Then with a suitable $k$-form, we have

$$
Z_{a}=\tau Z_{m}
$$

Remark. The point of Theorem 1-6 is that the condition (HW) is not necessary (see p.16 Remark in (Igusa [4])).

More explicitly, we can express Theorem 1-6 as follows.

Theorem 1-7. We have $Z_{a}(\omega, \Phi)=\tau Z_{m}(\omega, \Phi)$ for an irreducible regular $P . V$. which is castling-equivalent to one of the following reduced $P$. V.'s with the split $k$-form.

(1) $\left(H \times G L_{m}, \rho_{m}, M_{m}\right)$ where $H$ is any $k$-split connected semisimple algebraic subgroup of $S L_{m}$ with $\rho_{m}(h, g) x=h x^{t} g$ for $(g, h) \in H \times$ $G L_{m}$ and $x \in M_{m}$. We take a k-form $\left(H_{k} \times G L_{m}(k), \rho_{m}, M_{m}(k)\right)$. The relative invariant $f(x)=\operatorname{det} x$.

(2) $\left(G L_{2 m}, \rho, \mathrm{Alt}_{2 m}\right)$ where $\rho(g) x=g x^{t} g$ for $g \in G L_{2 m}$ and $x=$ $-{ }^{t} x \in \mathrm{Alt}_{2 m}$. We take a $k$-form $\left(G L_{2 m}(k), \rho, \mathrm{Alt}_{2 m}(k)\right)$. The relative invariant $f(x)=\operatorname{Pf}(x)$ (= the Pfaffian of $x$ ). 
(3) $\left(G L_{1} \times S O_{2 m}, \Lambda_{1} \otimes \Lambda_{1}, A f f^{2 m}\right)$ with $m \geq 2$.

Here $S O_{2 m}=\left\{A \in S L_{2 m},{ }^{t} A K A=K\right\}$ with $K=\left[\begin{array}{cc}0 & I_{m} \\ I_{m} & 0\end{array}\right]$ so that $f(x)=x_{1} x_{m+1}+\cdots+x_{m} x_{2 m}$ is the relative invariant. Let $G$ be the image of $G L_{1} \times S O_{2 m}$ by $\rho=\Lambda_{1} \otimes \Lambda_{1}$ in $G L_{2 m}$, and put $G_{k}=G \cap G L_{2 m}(k)$. We take a $k$-form $\left(G_{k}, k^{2 m}\right)$. For any $\lambda \in k^{\times}$, put

$$
g(\lambda)=\rho\left(\sqrt{\lambda},\left(\begin{array}{cc}
\sqrt{\lambda} I_{m} & 0 \\
0 & \frac{1}{\sqrt{\lambda}} I_{m}
\end{array}\right)\right)
$$

Then $g(\lambda)$ is in $G_{k}$ and $f(g(\lambda) x)=\lambda f(x)$.

Hence, with the $S O_{2 m}(k)$-homogeneity of $f^{-1}(1)$, we have $Y_{k}=G_{k} \cdot \xi$ with $\xi=e_{1}+e_{m+1}$. The isotropy subgroup $G_{\xi}$ of $G$ at $\xi$ is $S O_{2 m-1}$. Note that $\rho\left(-1,-I_{2 m}\right)=1$. Since $S O_{2 m-1}$ is connected, we have $Y_{A}=G_{A} \cdot \xi$ (cf. Theorem 1-8).

$(3)^{\prime} \quad\left(G L_{1} \times \operatorname{Spin}_{7}, \Lambda_{1} \otimes(\right.$ the spin rep. $\left.), V(8)\right)$

We identify $V(8)$ with $A f f^{8}$ by the standard base

$$
\left\{1, e_{i} e_{j}(1 \leqq i<j \leqq 4), e_{1} e_{2} e_{3} e_{4}\right\}
$$

Let $G$ be the image of $G L_{1} \times \operatorname{Spin}_{7}$ in $G L_{8}$ by $\Lambda_{1} \otimes$ (the spin rep.) and put $G_{k}=G \cap G L_{8}(k)$. We take a $k$-form $\left(G_{k}, k^{8}\right)$. Since the relative invariant is a quadratic form, we have $G \subset G O(8)$. By p.13 in (Igusa $[2])$, one sees that $Y_{k}=G_{k} \cdot \xi$. We have $Y_{A}=G_{A} \cdot \xi($ see Igusa [4]).

$(3)^{\prime \prime} \quad\left(G L_{1} \times \operatorname{Spin}_{9}, \Lambda_{1} \otimes \Lambda_{1}, V(16)\right)$

Everything is similar as $(3)^{\prime}$. In this case, we have $G \subset G O(16)$.

(4) $\left(S p_{m} \times G L_{2 r}, \Lambda_{1} \otimes \Lambda_{1}, M_{2 m, 2 r}\right)(m \geq 2 r)$

We take $k$-form

$$
\left(S p_{m}(k) \times G L_{2 r}(k), \Lambda_{1} \otimes \Lambda_{1}, M_{2 m, 2 r}(k)\right) .
$$

The relative invariant $f(x)=\operatorname{Pf}\left({ }^{t} x J x\right)$.

(5) $\left(G L_{1} \times E_{6}, \Lambda_{1}, \mathcal{J}(27)\right)$

$\mathcal{J}(27)$ is the totality of $3 \times 3$ hermitian matrices over the octonion algebra, and the relative invariant $f(x)$ is their determinant. The image $G$ of $G L_{1} \times E_{6}$ by $\Lambda_{1} \otimes \Lambda_{1}$ is $\operatorname{Sim}(f)$ and $G_{k}$ is transitive on $Y_{k}$ (see p.15 in (Igusa [2]).

(6) $\left(\operatorname{Spin}_{10} \times G L_{2},(\right.$ a half-spin rep. $\left.) \otimes \Lambda_{1}, V(16) \otimes V(2)\right)$ 
Let $G$ be the image of $\operatorname{Spin}_{10} \times G L_{2}$ in $G L_{32}$. We identify $V(16)$ with $A f f^{16}$ by the standard basis

$$
\left\{1, e_{i} e_{j}(1 \leqq i<j \leqq 5), e_{k}^{*}(1 \leqq k \leqq 5)\right\},
$$

and put $G_{k}=G L_{32}(k) \cap G$. Put $S_{i}(\lambda)=\lambda^{-1}+\left(\lambda-\lambda^{-1}\right) e_{i} e_{i+5}$ (see p.1002 in (Igusa [1])). For any $\alpha \in k^{\times}$, put $g(\alpha)=\left(s_{1}(\lambda) \cdots s_{5}(\lambda), \lambda I_{2}\right)$ with $\lambda={ }^{4} \sqrt{\alpha}$. Then we have $g(\alpha) \in G_{k}$ and $f(g(\alpha) x)=\alpha f(x)$. Since $f^{-1}(1)$ is $\operatorname{Spin}_{10}$-homogeneous, we can say that $Y_{\xi}=G_{k} \cdot \xi$ and $Y_{A}=$ $G_{A} \cdot \xi$ for

$(6)^{\prime} \quad\left(G L_{1} \times \operatorname{Spin}_{10}, \Lambda_{1} \otimes(\Lambda+\Lambda), V(16) \oplus V(16)\right)$ where $\Lambda$ is the (even) half-spin representation.

In particular, we have $Y_{k}=G_{k} \cdot \xi$ and $Y_{A}=G_{A} \cdot \xi$ for (6).

(7) $\left(G L_{7}, \Lambda_{3}, V(35)\right)$

Let $G$ be the image of $G L_{7}$ under $\Lambda_{3}$ in $G L_{35}$. For any local field $k \neq \mathbf{R}, Y_{k}$ is $G_{k}$-homogeneous and $Y_{\mathbf{R}}=G_{\mathbf{R}} \cdot \xi_{1} \sqcup G_{\mathbf{R}} \cdot \xi_{2}$. However we have \# $\left(G_{A} \backslash Y_{A}\right)<+\infty$. The relative invariant $f(x)$ is of degree 7 .

In this case, we have $Z_{a}=\tau Z_{m}$ by (Igusa [4]).

Theorem 1-8. Assume that a universally transitive regular $P$. V. $(G, V)$ defined over $k$ satisfies the two conditions:

(1) $Y_{k}=G_{k} \cdot \eta$

(2) the isotropy subgroup $G_{\eta}$ is connected.

Then we have $Z_{a}=\tau Z_{m}$.

Proof. By (2), every $G_{A}$-orbit in $Y_{A}$ contains a point of $Y_{k}$ (see (p.14 in Igusa [4]). Then, by (1), we have $Y_{A}=G_{A} \cdot \eta$. Hence by Proposition 1-2, we obtain our result.

Q.E.D.

\section{§3. Simple P.V.'s with \# $\left(G_{A} \backslash Y_{A}\right)<+\infty$}

Assume that \# $\left(G_{A} \backslash Y_{A}\right)<+\infty$ for a simple P.V. $(\tilde{G}, \rho, V)$ with $G=$ $\rho(\tilde{G})$. Then for almost all places $v$ of $k, Y_{v}$ must be $G_{v}$-transitive. Such non-irreducible regular simple P.V.'s with a semisimple generic isotropy subgroup $H=\rho\left(G_{\xi}\right)\left(\xi \in Y_{k}\right)$ are given as follows. (see (T. Kimura, S. Kasai and H. Hosokawa [8])).

(1) $\left(G L_{1} \times S L_{n}, \Lambda_{1} \otimes \Lambda_{1}+1 \otimes \Lambda_{1}^{*}\right)$ with $H=S L_{n}$.

(2) $(G L_{n}, \overbrace{\Lambda_{1} \oplus \cdots \oplus \Lambda_{1}}^{n})$ with $H=\{1\}$.

(3) $\left(G L_{1}^{n} \times G L_{n}, \rho_{n}+1 \otimes \Lambda_{1}\right)$ with $H=\{1\}$, where $\rho_{n}(g) x=$ $A x\left(\operatorname{diag}\left(\alpha_{1}, \cdots, \alpha_{n}\right)\right)$ for $g=\left(\alpha_{1}, \cdots \alpha_{n}, A\right) \in G L_{1}^{n} \times G L_{n}$ and $x \in M_{n}$. 
(4) $\left(G L_{1} \times S p_{n}, \Lambda_{1} \oplus\left(\Lambda_{1}+\Lambda_{1}\right)\right)$ with $H=S p_{n-1}$

(5) $\left(G L_{1} \times G L_{2 m}, 1 \otimes \Lambda_{2}+\Lambda_{1} \otimes\left(\Lambda_{1}^{(*)}+\Lambda_{1}^{(*)}\right)\right)$ with $H=S p_{m-1}$.

(6) $\left(G L_{2 m+1}, \Lambda_{2} \oplus \Lambda_{1}\right)$ with $H=S p_{m}$.

(7) $\left(G L_{1}^{3} \times G L_{2 m+1}, \Lambda_{2} \oplus \Lambda_{1} \oplus\left(\Lambda_{1} \oplus \Lambda_{1}\right)^{(*)}\right)$ with $H=S p_{m-1}$, where $G L_{1}^{3}$ acts on $\Lambda_{1} \oplus\left(\Lambda_{1} \oplus \Lambda_{1}\right)^{(*)}$ as scalar multiplications. Here $\left(\Lambda_{1} \oplus \Lambda_{1}\right)^{(*)}$ stands for $\Lambda_{1} \oplus \Lambda_{1}$ or its dual $\left.\left(\Lambda_{1} \oplus \Lambda_{1}\right)^{*}\right)$.

(8) $\left(G L_{1}^{2} \times \operatorname{Spin}_{n}\right.$, (a half-spin rep.) $\oplus$ (vector rep.) $)(n=8,10)$ with $H=\left(G_{2}\right)$ for $n=8$ and $H=\operatorname{Spin}_{7}$ for $n=10$.

(9) $\left(G L_{1} \times \operatorname{Spin}_{10}, \Lambda_{1} \otimes(\Lambda+\Lambda)\right)$ with $H=\left(G_{2}\right)$, where $\Lambda$ is the even half-spin representation.

We shall check each of them.

(1) We take a $k$-form $\left(G L_{1}(k) \times S L_{n}(k), \Lambda_{1} \otimes \Lambda_{1}+1 \otimes \Lambda_{1}^{*}, k^{n} \oplus k^{n}\right)$. Then $Y_{k}=G_{k} \cdot \xi$ with $\xi=\left(e_{1}, e_{1}\right)$ and $G_{\xi} \cong S L_{n-1}(k)$.

(2) For $(G L_{n}(k), \overbrace{\Lambda_{1} \oplus \cdots \oplus \Lambda_{1}}^{n}, M_{n}(k))$, we have $Y_{k}=G_{k} \cdot I_{n}$ and $H=\{1\}$.

(3) We take a $k$-form

$$
\left(G L_{1}^{n}(k) \times G L_{n}(k), M_{n}(k) \oplus k^{n}\right),
$$

and put $\xi=\left(I_{n},{ }^{t}(1, \cdots, 1)\right)$. Then $Y_{k}=G_{k} \cdot \xi$ and $G_{\xi}=\{1\}$.

(4) Let $\mathrm{G}$ be the image of $G L_{1} \times S p_{n}$ in $G L_{4 n}$ by $\rho=\Lambda_{1} \otimes\left(\Lambda_{1}+\Lambda_{1}\right)$, and put $G_{k}=G \cap G L_{4 n}(k)$. For any $\alpha \in k^{\times}$, put

$$
g(\alpha)=\left(\sqrt{\alpha},\left(\begin{array}{cc}
\sqrt{\alpha} I_{n} & 0 \\
0 & \frac{1}{\sqrt{\alpha}} I_{n}
\end{array}\right)\right) .
$$

Then $g(\alpha) \in G_{k}$ and $f(g(\alpha) x)=\alpha f(x)$. Since $f^{-1}(1)$ is $S p_{n}(k)$ transitive, we have $Y_{k}=G_{k} \cdot \xi$ with $\xi=\left(e_{1}, e_{n+1}\right)$ and $G_{\xi}=S p_{n-1}$ (see p.16 in [8]).

(5) We take a $k$-form

$\left(G L_{1}(k) \times G L_{2 m}(k), 1 \otimes \Lambda_{2}+\Lambda_{1} \otimes\left(\Lambda_{1}^{(*)}+\Lambda_{1}^{(*)}\right), \operatorname{Alt}_{2 n}(k) \oplus k^{2 m} \oplus k^{2 m}\right)$, 
where $\Lambda_{1}^{(*)}$ implies $\Lambda_{1}$ or its dual $\Lambda_{1}^{*}$. Since the generic isotropy subgroup of $\left(G L_{2 m}, \Lambda_{2}\right)$ is exactly $S p_{m}$, we have $Y_{k}=G_{k} \cdot \xi$ and $Y_{A}=G_{A} \cdot \xi$ by (4).

(6) Consider $\left(G L_{2 m+1}(k), \Lambda_{2} \oplus \Lambda_{1}, \operatorname{Alt}_{2 m+1}(k) \oplus k^{2 m+1}\right)$. Then $Y_{k}=G L_{2 m+1}(k) \cdot \xi$ with

$$
\xi=\left(\left[\begin{array}{ll}
J & 0 \\
0 & 0
\end{array}\right],{ }^{t}(0, \cdots, 0,1)\right)
$$

where $J=\left[\begin{array}{cc}0 & I_{m} \\ -I_{m} & 0\end{array}\right]$ and $G_{\xi}=S p_{m}(k)$. By Theorem 1-8, we have $Y_{k}=G_{k} \cdot \xi$ and $Y_{A}=G_{A} \cdot \xi$ for (6).

(7) We take a $k$-form

$$
\begin{aligned}
& \left(G L_{2 m+1}(k) \times G L_{1}^{3}(k), \Lambda_{2}+\Lambda_{1}+\left(\Lambda_{1}+\Lambda_{1}\right)^{(*)},\right. \\
& \left.\operatorname{Alt}_{2 m+1}(k) \oplus k^{2 m+1} \oplus k^{2 m+1} \oplus k^{2 m+1}\right)
\end{aligned}
$$

where $G L_{1}^{3}(k)$ acts on $k^{2 m+1} \oplus k^{2 m+1} \oplus k^{2 m+1}$ as scalar multiplications. Then we have $Y_{k}=G_{k} \cdot \xi$ with

$$
\xi=\left(\left(\begin{array}{ll}
J & 0 \\
0 & 0
\end{array}\right), e_{2 m+1}, e_{1}+e_{2 m+1}, e_{m+1}+e_{2 m+1}\right)
$$

and the image of the isotropy subgroup is connected. Note that $\left(-I_{2 m+1}\right.$, $-1,-1,-1)$ is in the kernel of

$$
\rho=\Lambda_{2} \oplus \Lambda_{1} \oplus\left(\Lambda_{1} \oplus \Lambda_{1}\right)^{(*)} .
$$

(8) Since the generic isotropy subgroup of $\left(\Lambda_{1} \otimes \phi\right)\left(G L_{1} \times \operatorname{Spin}_{2 n}\right)$ is $\phi\left(\operatorname{Spin}_{2 n-1}\right)$ where $\phi$ is the vector representation, we have $Y_{k}=G_{k} \cdot \xi$ and $Y_{A}=G_{A} \cdot \xi$ by using the results of irreducible case.

(9) In p.14 of (Igusa [2]), it is proved that $Y_{k}=G_{k} \cdot \xi$. One can see easily from p.11 of (Igusa [4]) that $G_{\xi}$ is connected so that $Y_{A}=G_{A} \cdot \xi$.

From the above observation,we obtain the following theorem.

Theorem 2-1. For a simple regular P.V. with \# $\left(G_{A} \backslash Y_{A}\right)<+\infty$, we have $Z_{a}=\tau Z_{m}$.

Theorem 2-2. For a simple regular P.V. with \# $\left(G_{A} \backslash Y_{A}\right)<+\infty$, we have $\#\left(G_{k} \backslash Y_{k}\right)=\#\left(G_{A} \backslash Y_{A}\right)=1$ for a suitable $k$-form. 


\section{§4. 2-Simple P.V.'s of Type I with \# $\left(G_{A} \backslash Y_{A}\right)<+\infty$}

By (T. Kimura, S. Kasai and H. Hosokawa [8]), all non-irreducible regular 2-simple P.V.'s of Type I with $\#\left(G_{A} \backslash Y_{A}\right)<+\infty$ are given as follows. Here we adjust the scalar multiplications so that the generic isotropy subgroup $H=\rho\left(G_{\xi}\right)$ is semisimple.

(1) $\left(G L_{1} \times G L_{5} \times G L_{2}, 1 \otimes \Lambda_{2} \otimes \Lambda_{1}+1 \otimes \Lambda_{1}^{*} \otimes 1+\Lambda_{1} \otimes \Lambda_{1}^{*} \otimes 1\right)$ with $H=\{1\}$.

(2) $\left(G L_{1} \times S p_{n} \times G L_{2 m}, 1 \otimes \Lambda_{1} \otimes \Lambda_{1}+\Lambda_{1} \otimes 1 \otimes\left(\Lambda_{1}^{(*)}+\Lambda_{1}^{(*)}\right)\right)$ $(n>m)$ with $H=S p_{n-m} \times S p_{m-1}$.

(3) $\left(S p_{n} \times G L_{2 m+1}, \Lambda_{1} \otimes \Lambda_{1}+\Lambda_{1} \otimes 1\right)(n>m)$ with $H=S p_{m} \times$ $S p_{n-m-1}$.

(4) $\left(G L_{1}^{3} \times S p_{n} \times G L_{2 m+1}, \Lambda_{1} \otimes \Lambda_{1}+\Lambda_{1} \otimes 1+1 \otimes\left(\Lambda_{1}+\Lambda_{1}\right)^{(*)}\right)(n>m)$ with $H=S p_{m-1} \times S p_{n-m-1}$, where $G L_{1}^{3}$ acts on $\Lambda_{1} \otimes 1+1 \otimes\left(\Lambda_{1}+\Lambda_{1}\right)^{(*)}$ as scalar multiplications.

(5) $\left(G L_{1} \times \operatorname{Spin}_{10} \times G L_{2}, 1 \otimes\right.$ (a half-spin rep.) $\otimes \Lambda_{1}+\Lambda_{1} \otimes 1 \otimes$ $\left.\left(\Lambda_{1}+\Lambda_{1}\right)\right)$ with $H=G_{2}$.

(6) $\left(G L_{1} \times \operatorname{Spin}_{10} \times G L_{1}^{2} \times G L_{2}, \Lambda_{1} \otimes\left(\right.\right.$ a half-spin rep.) $\otimes 1 \otimes \Lambda_{1}+$ $\left.1 \otimes 1 \otimes\left(\rho_{2}+1 \otimes \Lambda_{1}\right)\right)$ with $H=G_{2}$. (See (3) in $\S 3$ for $\left.\rho_{2}\right)$.

We shall check each of them.

(1) We take a $k$-form $\left(G L_{1}(k) \times G L_{5}(k) \times G L_{2}(k), 1 \otimes \Lambda_{2} \otimes \Lambda_{1}+1 \otimes \Lambda_{1}^{*} \otimes 1+\Lambda_{1} \otimes \Lambda_{1}^{*} \otimes 1\right)$.

Its generic isotropy subgroup is exactly $\{1\}$ (see (p.26-p.27 in T. Kimura, S. Kasai and H. Hosokawa [8]). We have $Y_{k}=G_{k} \cdot \xi$.

(2) We shall consider $\left(G L_{1} \times S p_{n} \times G L_{2 m}, 1 \otimes \Lambda_{1} \otimes \Lambda_{1}+\Lambda_{1} \otimes 1 \otimes\right.$ $\left.\left(\Lambda_{1}^{(*)}+\Lambda_{1}^{(*)}\right)\right)$

We take a $k$-form of the image of $\rho=1 \otimes \Lambda_{1} \otimes \Lambda_{1}+\Lambda_{1} \otimes 1 \otimes\left(\Lambda_{1}^{(*)}+\right.$ $\left.\Lambda_{1}^{(*)}\right)$.

Since $G L_{2 m}$-part of the generic isotropy subgroup of $\left(S p_{n} \times G L_{2 m}\right.$, $\left.\Lambda_{1} \otimes \Lambda_{1}\right)$ is $S p_{m}$, it reduces to (4) in $\S 3$.

(3) In this case, we have $G_{\xi}=S p_{m} \times S p_{n-m-1}$ and $Y_{k}=G_{k} \cdot \xi$ (cf. p.102 in (M. Sato and T. Kimura [5])). 
(4) $\left(\left(G L_{1}^{3} \times\right) S p_{n} \times G L_{2 m+1}, \Lambda_{1} \otimes \Lambda_{1}+\left(\Lambda_{1} \otimes 1+1 \otimes\left(\Lambda_{1}+\Lambda_{1}\right)^{(*)}\right)(n>\right.$ $m$ ) where $G L_{1}^{3}$ acts on $\Lambda_{1} \otimes 1+1 \otimes\left(\Lambda_{1}+\Lambda_{1}\right)^{(*)}$ as scalar multiplications. Since $G L_{2 m+1}$-part of the generic isotropy subgroup of $\left(G L_{1} \times S p_{n} \times\right.$ $\left.G L_{2 m+1}, 1 \otimes \Lambda_{1} \otimes \Lambda_{1}+\Lambda_{1} \otimes \Lambda_{1} \otimes 1\right)$ is $\left\{\left(\begin{array}{cc}A & 0 \\ 0 & \alpha\end{array}\right) ; A \in S p_{m} ; \alpha \in G L_{1}\right\}$, it reduces to (4) of $\S 3$. We have $Y_{k}=G_{k} \cdot \xi$ and $G_{\xi}=S p_{m-1} \times S p_{n-m-1}$. Note that $(-1)^{3} \times\left(-I_{2 n}\right) \times\left(-I_{2 m+1}\right)$ is in the kernel of $\rho=\Lambda_{1} \otimes \Lambda_{1}+$ $\Lambda_{1} \otimes 1+1 \otimes\left(\Lambda_{1}+\Lambda_{1}\right)^{(*)}$.

(5) $\left(G L_{1} \times \operatorname{Spin}_{10} \times G L_{2}, 1 \otimes \Lambda \otimes \Lambda_{1}+\Lambda_{1} \otimes 1 \otimes\left(\Lambda_{1}+\Lambda_{1}\right)\right)$ with $\Lambda=$ (a half-spin representation). Since the generic isotropy subgroup of $\left(G L_{1} \times G L_{2}, \Lambda_{1} \otimes\left(\Lambda_{1}+\Lambda_{1}\right)\right)$ is $\left\{\left(\alpha^{-1}, \alpha I_{2}\right) ; \alpha \in G L_{1}\right\}$, (5) reduces to (9) in $\S 3$.

(6) Since $G L_{2}$-part of the generic isotropy subgroup of $\left(G L_{1}^{2} \times\right.$ $\left.G L_{2}, \rho_{2}+1 \otimes \Lambda_{1}\right)$ is 1 (see $(3)$ in $\S 3$ ), reduces to (9) in $\S 3$.

Theorem 3-1. For a regular 2-simple P.V. of type I with $\#\left(G_{A} \backslash Y_{A}\right)<+\infty$, we have $Z_{a}=\tau Z_{m}$.

Theorem 3-2. For a regular 2-simple P.V.'s of type I with $\#\left(G_{A} \backslash Y_{A}\right)<+\infty$, we have \# $\left(G_{k} \backslash Y_{k}\right)=\#\left(G_{A} \backslash Y_{A}\right)=1$ for a suitable $k$-form.

\section{References}

[1] J. Igusa, A classification of spinors up to dimension twelve, Amer. J. Math., 92 (1970), 997-1028.

[2] _ On functional equations of complex powers, Invent. Math., 85 (1986), 1-29.

[3] On a certain class of prehomogeneous vector spaces, J. Pure Appl., 47 (1987), 265-282.

[4] Z Zeta distributions associated with some invariants, Amer. J. Math., 109 (1987), 1-34.

[5] M. Sato and T. Kimura, A classification of irreducible prehomogeneous vector spaces and their relative invariants, Nagoya Math. J., 65 (1977), $1-155$.

[6] T. Kimura, A classification of prehomogeneous vector spaces of simple algebraic groups with scalar multiplications, J. Algebra, 83 No. 1 (1983), 72-100.

[7] T. Kimura, S. Kasai, M. Inuzuka and O. Yasukura, A classification of 2-simple prehomogeneous vector spaces of type I, J. Algebra, 114 No. 2 (1988), 369-400. 
[8] T. Kimura, S. Kasai and H. Hosokawa, Universal transitivity of simple and 2-simple prehomogeneous vector spaces, Ann. Inst. Fourier (Grenoble), 38,2 (1988), 11-41.

[9] F. Sato, Zeta functions in several variables associated with prehomogeneous vector spaces II: A covergence criterion, Tohoku Math. J., 35 No. 1 (1983), 77-99.

[10] T. Kimura, The $b$-functions and holonomy diagrams of irreducible regular prehomogeneous vector spaces, Nagoya Math. J., 85 (1982), 1-80.

[11] _ Iwasawa-Tate theory for prehomogeneous vector spaces with $Z a=\tau Z m$.

[12] A. Borel and Harish-Chandra, Arithmetic subgroups and Algebraic groups, Ann. of Math., 75 (1962), 458-535.

[13] T. Ono, an integral attached to a hypersurface, Amer. J. Math., 90 (1968), 1224-1236.

The Institute of Mathematics

University of Tsukuba

Ibaraki, 305

Japan 